\title{
Índice ceo-d y su relación con la calidad de vida en la salud oral de preescolares de la I.E. Cesar Vallejo de Chorrillos, junio 2018
}

\author{
Rocha Lerzundi Joel Maycol*1,a; Gómez Gonzales Walter Edgar 2,b; Bernardo Santiago Grisi 1,c
}

RESUMEN

Objetivo: Determinar la relación entre el índice ceo-d y la calidad de vida en la salud oral (CVRSO) de preescolares de la I.E. Cesar Vallejo de Chorrillos - 2018.

Materiales y métodos: Estudio observacional y transversal que evaluó a 118 niños de 3 a 5 años. Se registró la experiencia de caries dental con el índice ceo-d y la encuesta ECOHIS para la percepción de la calidad de vida en salud oral. Las pruebas estadísticas empleadas fueron t de Student, Kruskal-Wallis y U Mann-Whitney, con nivel de significancia 0,05; y coeficiente Spearman 0,01.

Resultados: El promedio del índice ceo-d $(6,60 \pm 3,95)$ indica un nivel de severidad muy alto y frecuencia $88,98 \%$. El ECOHIS reflejó un impacto negativo en la calidad de vida en salud oral $(21,72 \pm 14,15)$, y una alta frecuencia $(85,59 \%)$; la dimensión más frecuente fue limitación funcional 83,90\%. Según el coeficiente de Spearman, existe una relación directa positiva moderada significativa $(r=0,682)$ entre las variables caries dental y calidad de vida; y demuestra en esta relación que su predominio radica en el sexo femenino con $21,83 \pm 12,56$, y el grupo de 5 años que obtuvo $33,67 \pm 11,17$, ambos estadísticamente significativos $(p=0,000)$.

Conclusiones: La caries dental genera un impacto negativo en la calidad de vida y afecta las diversas actividades de los niños, así como también, repercute en su familia y su entorno. Al haber un incremento del número de caries dental y su severidad, habrá un mayor impacto negativo en la calidad de vida, que se agrava a mayor edad y que tiene una mayor predisposición en las niñas.

Palabras clave: Caries dental; Calidad de vida; Salud bucal; Preescolar (Fuente: DeCS BIREME).

\section{The ceo-d index and its relationship with oral health-related quality of life in preschool children of the I.E. César Vallejo, Chorrillos, june 2018}

ABSTRACT

Objective: To determine the relationship between the ceo-d index and the oral health-related quality of life (OHRQoL) in preschool children of the I.E. César Vallejo, Chorrillos - 2018.

Materials and methods: An observational and cross-sectional study, which evaluated 118 children aged 3 to 5 who registered dental caries according to the ceo-d index and the ECOHIS survey for the perception of oral health-related quality of life. The statistical tests used in the research were the Student's t test, Kruskal-Wallis $\mathrm{H}$ test and Mann-Whitney $\mathrm{U}$ test, with a significance level of 0.05 and a Spearman coefficient of 0,01 .

Results: The average of the ceo-d index was 6,60 $\pm 3,95$, which indicates a very high level of severity, and a frequency of $88.98 \%$. The ECOHIS survey showed a negative impact $(21,72 \pm 14,15)$ and a high frequency $(85.59 \%)$ on the oral healthrelated quality of life. The most frequent dimension was "functional limitation" (83.90 \%). According to the Spearman coefficient, there is a significant moderate positive direct relationship $(r=0,682)$ between the variables "dental caries" and "quality of life". This relationship prevails in the female $(21.83 \pm 12.56)$ and 5-year-old $(33.67 \pm 11.17)$ groups, , both of which were statistically significant $(p=0.000)$.

Conclusions: Dental caries generate a negative impact on the quality of life that affects the various activities of children, and has repercussions on their family and environment. The larger the number of dental caries and severity, the greater negative impact on the quality of life. This condition worsens with increasing age, and girls are more prone to have it.

Keywords: Dental caries; Quality of life; Oral Health; Child, Preschool (Source: MeSH NLM).

1. Universidad Privada Telesup. Lima, Perú.

2. Universidad Nacional Mayor de San Marcos. Lima, Perú.

a. Bachiller en Odontología.

b. Doctor en Ciencias de la Salud.

c. Directora de Investigación Tecnológica.

* Autor corresponsal. 


\section{INTRODUCCIÓN}

La boca cumple un papel importante en la vida cotidiana del niño. Desde los primeros años, aprende y percibe el mundo por medio del vínculo oroambiental, y estimula su propio crecimiento y desarrollo, estilo de vida y hábitos que lo ayudarán en sus actividades diarias en el futuro. Estas funciones conllevan a que el ser humano tenga una gran interacción con sí mismo y con el medio que lo rodea.

La caries dental $(C D)$, es una enfermedad infecciosa producida por una alteración del equilibrio en el ecosistema oral. Cuando este proceso dinámico entre la desmineralización y la remineralización falla, la acción de los carbohidratos fermentables originan, con el tiempo, el reblandecimiento de los cristales de hidroxiapatita del esmalte dental y la desintegración de la estructura orgánica de la pieza dentaria (1); es la enfermedad de mayor prevalencia a nivel mundial.

Esta afección se puede presentar en las etapas iniciales de la vida; si no recibe la debida atención por parte de los padres, trae consecuencias en la salud oral y en el estado general del niño; la más relevante de todas, la pérdida dental prematura que lleva a la aparición de alteraciones como maloclusiones, apiñamiento dental, defectos óseos, inflamación gingival, etc., y lo condiciona, en el futuro, a presentar sobrecarga masticatoria que originará dolor, inflamación y baja funcionalidad. La ausencia de piezas dentales tendrá repercusiones estéticas que producirán un impacto directo en la interrelación del niño con su entorno social, lo que dará, como resultado, una baja autoestima.

La primera edición del Atlas de salud bucodental, publicado en 2009 , tenía por objetivo "dibujar el mapa del abandono de la salud bucodental". La extensión del problema no ha cambiado, sin embargo, surgen nuevas y prometedoras oportunidades para el enfoque a escala global de las enfermedades bucodentales ${ }^{(2)}$.

Actualmente, el Perú presenta una prevalencia de caries de un $98 \%$, y continúa la aparición de nuevos casos. En Lima, 12 de cada 100 personas presentan la lesión dental (reportado en 2009 y 2010) ${ }^{(2)}$.

Así también, Torres $\mathrm{H}$ reportó $83,3 \%$ de casos en niños de 3 años y $87 \%$ en un grupo de 4 y 5 años ${ }^{(3)}$.

Villena, en un estudio de 2011, encontró que la prevalencia de $C D$ en niños menores de 71 meses en Lima fue $62,3 \%$, y que se incrementa con la edad: $10,5 \%(0$ 11 meses), 27,3 \% (12-23 meses), 60,0 \% (24-35 meses), $65,5 \%$ (36-47 meses), 73,4\% (48-59 meses) y 86, 9 \% (6071 meses); además, el índice ceo-d promedio fue 2,97 , donde el componente cariado representó el 99,9\% del mismo ${ }^{(4)}$.

El ceo-d fue elaborado por Allen Gruebbel en 1944, como complemento del índice CPO que tabula los dientes cariados, perdidos y obturados en la población adulta; al elaborarlo y surgir posibilidad de confundirse las letras CPO propuso la nueva simbología dispuesta para la población infantil (5), por medio de un proceso sistemático, que codifica como cariados, extraídos y obturados en dientes deciduos que presenten lesiones de caries, los cuales cuantificaron con este índice la experiencia de tal patología y determinaron el nivel de severidad con los intervalos de siguientes: ceo-d $(0-1,2)=$ muy bajo, $(1,3-2,6)=$ bajo, $(2,7-4,4)=$ moderado, $(4,5$ $-6,5)=$ alto y $(>6,5)=$ muy alto ${ }^{(6,7)}$.

El dolor es uno de los factores reportados que causa bajo rendimiento laboral, frustración, estrés y ansiedad en las personas adultas y, además, puede traer mayor perjuicio en los niños, porque ocasiona depresión, aislamiento, ausentismo escolar, desconcentración, insomnio y también alteraciones en el comportamiento $(6,8)$, lo que relaciona la CD a otros problemas orales y generales.

Las alteraciones en el niño pueden darse a nivel funcional (perdida dental/dolor), estético (sonreír), psicológico (autoestima), entre otros, lo que puede repercutir en su entorno familiar (estrés) y escuela (ausentismo escolar), lo que impacta de forma negativa en su calidad de vida (CV) en salud oral.

La calidad de vida (CV) es un estado de satisfacción física, psicosocial, y no únicamente de la carencia de afecciones o enfermedades ${ }^{(9)}$. Al ser un concepto multidimensional, multifactorial y evolutivo, de acuerdo al contexto en el que se sitúa el individuo y según su percepción, la cual es subjetiva, única, puede variar en cada ámbito de su vida, como también, en el transcurso de la misma ${ }^{(10)}$. La calidad de vida relacionada a salud oral (CVRSO) es conceptualizada por la OMS como ausencia de dolor en la boca y cara, cáncer oral $\mathrm{y} / \mathrm{o}$ de orofaringe, infecciones, heridas bucales, enfermedad del periodonto, caries, ausencia del órgano dental, entre otros trastornos y síndromes $(6,11)$. 
Adulyanon y Sheiham (1997) propusieron 3 niveles para explicar los efectos de las condiciones orales en las actividades cotidianas. El nivel 1 muestra el estado bucal (capacidad física); el nivel 2 se refiere a los impactos negativos a causa del malestar oral como incomodidad, limitación funcional y disconfort de su imagen (capacidad psicológica); finalmente, el nivel 3 registra los impactos que interfieren en actividades diarias (capacidad social) ${ }^{(12)}$.

La encuesta ECOHIS fue creada por Talekar Pahel y sus colaboradores en 2007 en Carolina del Norte, E.E. U.U. $(6,13)$. Se compone de 13 preguntas, distribuidas en 2 segmentos; el primero es impacto sobre el niño, que evalúa los síntomas orales, limitaciones funcionales, aspectos psicológicos, autoimagen/interacción social; el segundo, el impacto sobre la familia, que evalúa la angustia de los padres y función familiar, con un rango de valor final de 0-52 pts. ${ }^{(6,13)}$, donde a mayor sea la sumatoria, mayor será el impacto negativo en la CV en la salud oral del menor. Ha sido traducido y adaptado a varios idiomas, entre ellos, el español "peruano", y aplicado en varios estudios publicados por ser válido y confiable (14-17), con un Alfa de Cronbach de 0,925 en el impacto en el niño, 0,882 impacto sobre la familia, y una puntuación general de $0,948^{(16)}$.

Actualmente, en nuestro país, no tenemos investigaciones suficientes aplicadas en este grupo etario que relacionen estos dos conceptos; por lo tanto, este trabajo busca incentivar y marcar un camino a otras investigaciones con el grupo infantil a futuro, considerado como una población vulnerable; por ello, la finalidad de este estudio es determinar la relación que se presenta entre el índice ceo-d para cuantificar la historia dental, y el impacto en la CV en salud oral medido con la encuesta ECOHIS (Early Childhood Oral Health Impact Scale), con lo que se obtiene un diagnóstico actual de cómo este problema afecta en otros aspectos personales de la población.

\section{MATERIALES Y MÉTODOS}

\section{Diseño y población de estudio}

El presente trabajo es un estudio observacional, transversal con enfoque cuantitativo y correlacional (18). La población fue de 266 infantes de 3 a 5 años de la I.E pública mixta Cesar Vallejo, ubicada en el distrito de Chorrillos. El cálculo muestral se realizó mediante la fórmula para población finita, se utilizó un cálculo para estimar una media, que dio una muestra de 118 niños seleccionados por medio de la tecnica de muestreo probabilístico estratificado proporcional homogenizado de acuerdo a su edad y sexo.

\section{Variables y mediciones}

Las variables a estudiar fueron el Índice ceo-d y calidad de vida en la salud oral. Se aplicó el cuestionario ECOHIS, una encuesta multidimensional de forma estructurada, dirigida a los padres para evaluar la calidad de vida en relación con la calidad de vida de sus menores. Además, se realizó a los niños una inspección clínica visual oral (bajo criterios establecidos por la OMS en su Manual Oral Health Surveys Basic Methods ${ }^{(7)}$, normas éticas y de bioseguridad), que se registró en el odontograma para identificar y graficar las alteraciones de los dientes deciduos, con los fundamentos que se estipulan en la norma técnica, ley $N^{\circ} 15251$ que crea al Colegio Odontológico del Perú modificada por Ley $29016^{(19,20)}$. Finalmente, se cuantificó la caries dental y su nivel de severidad en los dientes deciduos de acuerdo al índice ceo-d ${ }^{(5)}$.

\section{Análisis estadístico}

El procesamiento de los datos se realizó mediante el programa estadístico SPSS versión 25 , se utilizó la prueba estadística paramétrica $t$ de Student, para identificar al índice ceo-d según el sexo del niño y otras no paramétricas como la $\mathrm{H}$ de Kruskal-Wallis y la U de Mann-Whitney, para hallar ambas variables según la edad del niño. Para la contrastación de la hipótesis se utilizó el coeficiente de correlación de Spearman; se aceptó como significativo el valor $p<0,05$, que nos garantiza la calidad de la relación, autenticidad y veracidad de los datos.

\section{Consideraciones éticas}

Para evitar transgredir los derechos de los participantes y mantener en reserva los datos recolectados y procedimientos en la evaluación, se explicó el procedimiento verbalmente al padre. Se aplicó el consentimiento informado bajo principios fundamentales de bioética como son autonomía, beneficencia, justicia y no maleficencia ${ }^{(21)}$.

\section{RESULTADOS}

Los resultados obtenidos con el índice ceo-d al medir el nivel de severidad y frecuencia de $C D$, muestran un $49,15 \%$ de niños que presentan un nivel muy alto; mientras que el $20,34 \%$, un nivel alto, y $5,93 \%$, un nivel bajo. El promedio total del índice ceo-d fue de $6,60 \pm 3,95$, lo que indica un grado de severidad de CD muy alto. El 88,98 \% de niños presentan CD (Tabla 1). 
Tabla 1. Índice del ceo-d de los preescolares de la I.E. Cesar Vallejo de Chorrillos - 2018

\begin{tabular}{|ccc|}
\hline Nivel de severidad & $\mathbf{n}$ & $\%$ \\
\hline MUY BAJO & 12 & 10,17 \\
BAJO & 7 & 5,93 \\
MODERADO & 17 & 14,41 \\
ALTO & 24 & 20,34 \\
MUY ALTO & $\mathbf{5 8}$ & $\mathbf{4 9 , 1 5}$ \\
\hline Experiencia de caries & 105 & 88,98 \\
\hline Índice ceo-d & Media \pm DE & $\mathbf{6 , 6 0} \pm \mathbf{3 , 9 5}$ \\
\hline
\end{tabular}

DE: Desviación estándar.

La calificación del ECOHIS de acuerdo a sus dimensiones con sus puntajes, promedios y frecuencias muestra una máxima de 47 puntos, con promedio total 21,72 $\pm 14,15$, lo que evidencia un impacto negativo en la $\mathrm{CV}$ en salud oral infantil. La sección "impacto en el niño", dio un resultado $4,40 \pm 9,92$; y la dimensión “limitación funcional" fue la que presentó el promedio más elevado $6,49 \pm 4,38$; seguida por "autoimagen/ interacción" con 2,81 $\pm 2,42$; mientras que, en "impacto en la familia" se obtuvo 7,31 $\pm 4,62$, y, dentro de esta, la dimensión "angustia de los padres" tuvo el promedio más alto $(3,72 \pm 2,36)$, lo que refleja un impacto negativo en la CV en salud oral del menor. Las dimensiones de mayor frecuencia fueron "limitaciones funcionales" con 83,90\% de preescolares afectados, "síntomas orales" con 83,05\%; y "angustia de los padres" con $84,75 \%$ dentro de la sección del "impacto en la familia" (Tabla 2).

Tabla 2. Nivel de calidad de vida en la salud oral de los preescolares de la I.E. Cesar Vallejo de Chorrillos - 2018

\begin{tabular}{|c|c|c|c|c|c|c|c|}
\hline \multirow[b]{2}{*}{$\begin{array}{l}\text { Dimensiones } \\
\text { del ECOHIS }\end{array}$} & \multirow[b]{2}{*}{$\begin{array}{l}\text { Número de } \\
\text { preguntas }\end{array}$} & \multicolumn{5}{|c|}{ Calidad de vida en la salud oral (CVRSO) } & \multirow[b]{2}{*}{$\begin{array}{c}\text { Rango del } \\
\text { ECOHIS }\end{array}$} \\
\hline & & Media (\%) & DE & Mín. & Máx. & $\begin{array}{l}\text { Rango } \\
\text { obtenido }\end{array}$ & \\
\hline Puntuación total & 13 & $21,72(85,59)$ & 14,15 & 0 & 47 & $0-47$ & $0-52$ \\
\hline \multicolumn{8}{|l|}{ Dimensiones } \\
\hline Impacto en el niño & 9 & $14,40(84,75)$ & 9,92 & 0 & 33 & $0-33$ & $0-36$ \\
\hline Síntomas orales & 1 & $2,46(83,05)$ & 1,45 & 0 & 4 & $0-4$ & $0-4$ \\
\hline Limitaciones funcionales & 4 & $6,49(83,90)$ & 4,38 & 0 & 15 & $0-15$ & $0-16$ \\
\hline Alteraciones psicológicas & 2 & $2,67(74,58)$ & 2,24 & 0 & 8 & $0-8$ & $0-8$ \\
\hline Autoimagen/interacción social & 2 & $2,81(68,64)$ & 2,42 & 0 & 8 & $0-8$ & $0-8$ \\
\hline Impacto en la familia & 4 & $7,31(85,59)$ & 4,62 & 0 & 16 & $0-16$ & $0-16$ \\
\hline Angustia de los padres & 2 & $3,72(84,75)$ & 2,36 & 0 & 8 & $0-8$ & $0-8$ \\
\hline Función familiar & 2 & $3,58(82,20)$ & 2,36 & 0 & 8 & $0-8$ & $0-8$ \\
\hline
\end{tabular}

DE: Desviación estándar.

Se halló que la relación entre el ceo-d y la CV en salud oral, donde los preescolares con un índice ceo-d mayor de 6,5 presentan mayor promedio de 30,24 $\pm 11,11$ con relación al promedio total del ECOHIS; en comparación con el ceo-d menor a 1,2, con un promedio de 0,58 $\pm 2,02$. Hay diferencia estadísticamente significativa entre los valores promedios del ECOHIS y el índice ceo-d (prueba $\mathrm{H}$ de Kruskall Wallis; $\mathrm{p}<0,05$ ). Según el coeficiente de Spearman, existe una relación directa positiva moderada entre el índice ceo-d y la CV en relación a la salud oral $(r=0,682)$, lo que es estadísticamente significativo $(p=0,000)$ (Tabla 3$)$. 
Tabla 3. Relación entre el índice ceo-d y la calidad de vida en la salud oral (CVRSO) de preescolares de la I.E. Cesar Vallejo de Chorrillos

\begin{tabular}{ccccccc} 
& \multicolumn{7}{c}{ Calidad de vida en la salud oral (CVRSO) } \\
\cline { 2 - 7 } Índice ceo-d & $\mathbf{n}$ & $\%$ & Media & DE & $\mathbf{r}$ (Spearman) & Valor $\mathbf{p}$ \\
\hline Muy bajo & 12 & 10,17 & 0,58 & 2,02 & & \\
Bajo & 7 & 5,93 & 11,71 & 8,67 & & $\mathbf{0 , 0 0 0}$ \\
Moderado & 17 & 14,41 & 13,76 & 13,40 & $\mathbf{0 . 6 8 2} * *$ & \\
Alto & 24 & 20,34 & 20,25 & 8,43 & & \\
Muy alto & 58 & 49,15 & 30,24 & 11,11 & & \\
\hline
\end{tabular}

Prueba $\mathrm{H}$ de Kruskal Wallis; $\mathrm{p}<0,05$.

DE: Desviación estándar.

${ }^{* *}$ Coeficiente Spearman: La correlación es significativa en el nivel 0,01 (bilateral).

Se observa que en la correlación entre el índice ceo-d y la CV en relación a la salud oral según el sexo de los preescolares, el sexo femenino obtuvo un promedio ligeramente mayor de $(21,83 \pm 12,56)$ en comparación con el masculino $21,60 \pm 15,74$, este hallazgo es estadísticamente significativo $(p=0,000)$. En el análisis con el coeficiente de correlación de Spearman, se observa que existe una relación positiva moderada fuerte entre el índice ceo-d y la CV en relación a la salud oral de la muestra evaluada según el sexo del preescolar $(r=0,689)$, Lo que es estadísticamente significativo $(p=0,000)$ (Tabla 4$)$.

Tabla 4. Relación entre el índice ceo-d y la calidad de vida en la salud oral (CVRSO) de preescolares de la I.E. Cesar Vallejo de Chorrillos según el sexo de los preescolares

\begin{tabular}{|c|c|c|c|c|c|c|c|}
\hline \multirow[b]{2}{*}{ Sexo } & \multirow[b]{2}{*}{ Índice ceo-d } & \multicolumn{6}{|c|}{ Calidad de vida en la salud oral (CVRSO) } \\
\hline & & $\mathbf{n}$ & $\%$ & Media & DE & $\mathbf{r}$ (Spearman) & Valor $p$ \\
\hline & Muy bajo & 8 & 13,79 & 0,87 & 2,48 & & \\
\hline & Bajo & 2 & 3,45 & 6,50 & 9,19 & & \\
\hline \multirow[t]{6}{*}{ Masculino } & Moderado & 7 & 12,07 & 11,57 & 15,21 & $0,689 * *$ & 0,000 \\
\hline & Alto & 10 & 17,24 & 21,80 & 7,83 & & \\
\hline & Muy alto & 31 & 53,45 & 30,13 & 13,18 & & \\
\hline & Total & 58 & $100 \%$ & 21,60 & 15.74 & & \\
\hline & Muy bajo & 4 & 6,67 & 0,00 & 0,00 & & \\
\hline & Bajo & 5 & 8,33 & 13,80 & 8,53 & & \\
\hline \multirow[t]{4}{*}{ Femenino } & Moderado & 10 & 16,67 & 15,30 & 12,60 & $0,689 * *$ & 0,000 \\
\hline & Alto & 14 & 23,33 & 19,14 & 8,94 & & \\
\hline & Muy alto & 27 & 45,00 & 30,37 & 8,37 & & \\
\hline & Total & 60 & $100 \%$ & 21,83 & 12,56 & & \\
\hline
\end{tabular}

DE: Desviación estándar.

**Coeficiente Spearman: La correlación es significativa en el nivel 0,01.

Respecto a la correlación entre el índice ceo-d y la $\mathrm{CV}$, relacionados a la salud oral, según la edad de los preescolares, el grupo de 5 años obtuvo un mayor promedio $(33,67 \pm 11,17)$, en comparación con los de 4 años $(22,52 \pm 9,80)$ y 3 años $(9,47 \pm 10,49)$, y es estadísticamente significativa $(\mathrm{p}=0,000)$. Además, en el nivel de muy alta severidad, los niños de 5 años mostraron un mayor promedio de $39,76 \pm 5,17$, sobre los niños de 4 años $(26,68 \pm 9,47)$ y los de 3 años $(21,00$ $\pm 10,00$ ). Según el análisis de coeficiente de correlación 
de Spearman, se observa que existe una relación positiva moderada fuerte entre el índice ceo-d y la $\mathrm{CV}$ en relación a la salud oral de la muestra evaluada según la edad de preescolar $(r=0,709)$, lo que también es estadísticamente significativo $(p=0,000)$ (Tabla 5$)$.

Tabla 5. Relación entre el índice ceo-d y la calidad de vida en la salud oral (CVRSO) de preescolares de la I.E. Cesar Vallejo de Chorrillos según la edad de los preescolares

\begin{tabular}{|c|c|c|c|c|c|c|c|}
\hline \multirow[b]{2}{*}{ Edad } & \multirow[b]{2}{*}{ Índice ceo-d } & \multicolumn{6}{|c|}{ Calidad de vida en la salud oral (CVRSO) } \\
\hline & & $\mathbf{n}$ & $\%$ & Media & DE & r (Spearman) & Valor $p$ \\
\hline & Muy bajo & 8 & 21,05 & 0,87 & 2,48 & & \\
\hline & Bajo & 2 & 5,26 & 0,00 & 0,00 & & \\
\hline \multirow[t]{6}{*}{3 años } & Moderado & 10 & 26,32 & 4,50 & 4,77 & $0,709^{\star *}$ & 0,000 \\
\hline & Alto & 6 & 15,79 & 9,33 & 4,63 & & \\
\hline & Muy alto & 12 & 31,58 & 21,00 & 10,00 & & \\
\hline & Total & 38 & $100 \%$ & 9,47 & 10,49 & & \\
\hline & Muy bajo & 2 & 4,55 & 0,00 & 0,00 & & \\
\hline & Bajo & 4 & 9,09 & 14,75 & 2,06 & & \\
\hline \multirow[t]{6}{*}{4 años } & Moderado & 3 & 6,82 & 20,00 & 0,00 & $0,709^{\star *}$ & 0,000 \\
\hline & Alto & 10 & 22,73 & 20,50 & 4,95 & & \\
\hline & Muy alto & 25 & 56,82 & 26,68 & 9,47 & & \\
\hline & Total & 44 & $100 \%$ & 22,52 & 9,80 & & \\
\hline & Muy bajo & 2 & 5,56 & 0,00 & 0,00 & & \\
\hline & Bajo & 1 & 11,11 & 23,00 & 0,00 & & \\
\hline \multirow[t]{4}{*}{5 años } & Moderado & 4 & 2,78 & 32,25 & 10,44 & $0,709^{\star *}$ & 0,000 \\
\hline & Alto & 8 & 22,22 & 28,13 & 3,64 & & \\
\hline & Muy alto & 21 & 58,33 & 39,76 & 5,17 & & \\
\hline & Total & 36 & $100 \%$ & 33,67 & 11,17 & & \\
\hline
\end{tabular}

DE: Desviación estándar

${ }^{* *}$ Coeficiente Spearman: La correlación es significativa en el nivel 0,01.

\section{DISCUSIÓN}

El estudio abarcó uno de los problemas orales que afecta gran parte de la población, principalmente a los preescolares, como es la CD, de la que se obtuvo una alta frecuencia de $88,98 \%$, lo que coincide con los estudios de Quispe M y Quintana C ${ }^{(14)}$, que reportaron que el $74 \%$ de preescolares tienen CD en Lima, Perú; Bansal $P$ et al. ${ }^{(22)}$, con $85 \%$ de frecuencia de $C D$ en niños de 2 a 5 años en la India; a su vez, Alosaimi B et al. (23), informaron que en la población de su estudio (niños de 3 a 5 años en Riyadh, Arabia Saudita) obtuvieron una frecuencia de $85,5 \%$. Así mismo, Zambrano 0 et al. (24), mostraron que los niños en edad preescolar en Zulia (Venezuela) tienen una frecuencia de CD de $79,5 \%$; Bagińska J et al. ${ }^{(25)}$, encontraron que el nivel de $C D$ es de $85,9 \%$, afectaba a los niños de 5 años de edad en Polonia; López R y García C (16), reportaron que la $C D$ presenta una frecuencia de $76,47 \%$, en niños de 3 a 5 años en Lima, Perú. Por otro lado, algunos estudios evidencian distintos resultados a los obtenidos, causados por el descuido parental, dieta del menor y falta de inversión en gestión de políticas para programas de prevención y promoción de salud en esta población. Así tenemos el trabajo de Kamran $\mathrm{R}$ et al. (26), que muestra un 3,09\% de $C D$ en los niños de 4 y 5 años de Pakistán; Tello $G$ et al. (15) encontraron que la $C D$ en niños de 3 y 4 años, tenían una prevalencia de 33,1 \% en Sao Paulo, Brasil; y Duarte I ${ }^{(6)}$, encontró un 20,3 \% de afección de la CD en los preescolares de 3 a 5 años en Cascáis, Portugal.

De acuerdo al nivel de severidad de CD propuesto por 
la OMS, el promedio del índice ceo-d de la población de este estudio fue de 6,60 $\pm 3,95$, que lo ubica en un nivel muy alto. Estos datos son similares a los reportados por Bansal $P$ et al. (22), que evalúa a niños de 2 a 5 años, y obtiene un promedio de $7,13 \pm 5,24$, con nivel muy alto en la India; Abanto J et al. (27) hallaron un nivel muy alto con promedio ceo-d de 7,29 $\pm 2,78$, en niños de 2 a 5 años en Sao Pablo, Brasil; Bagińska J et al. ${ }^{(25)}$, encontraron un nivel alto con promedio $5,56 \pm 4,45$ en niños de 5 años en Polonia; Alosaimi B et al. ${ }^{(23)}$, identificaron un nivel de severidad alto, con un promedio de ceo-d 5,54 $\pm 3,49$ en niños de 3 a 5 años en Riyadh, Arabia Saudita. Otros grupos de investigadores informaron distintos promedios, que son menores a los obtenidos; los cuales pueden estar asociados principalmente al desinterés y desconocimiento de los padres, además de la limitación por el nivel socioeconómico familiar. Tello $\mathrm{G}$ et al. ${ }^{(15)}$, muestran un nivel moderado con promedio de 3,07 de índice ceo-d en niños de 3 y 4 años en Sao Paulo, Brasil; Patil $S$ et al. ${ }^{(28)}$, hallaron un nivel bajo $(2,57 \pm 0,56)$ en niños de 3 a 5 años en Karad, India; Mehta A y Bhalla $S{ }^{(29)}$, encontraron un nivel bajo con promedio de 2,54 $\pm 2,4$ en niños de 5 a 6 años en India; Kamran $R$ et al. (26), obtuvieron un nivel muy bajo con promedio de $1,04 \pm .0,23$ en niños de 4 a 5 años, en Pakistán; por último, Duarte I ${ }^{(6)}$ identificó en su población un nivel muy bajo con 0,71 de promedio, en niños de 3 a 5 años en Cascais, Portugal.

Cuando se habla sobre la CV en salud oral (CVRSO) de preescolares se evalúa el impacto que tienen los problemas orales sobre esta, la cual, en esta investigación obtuvo un valor máximo de 47 puntos, con promedio de $21,72 \pm 14,15$, que representa un mayor impacto negativo en la CV en salud oral de los niños; lo que coincide con Mamani $V$ y Padilla $T$ (30), que encontraron un valor de $24,9 \pm 8,3$ en niños de 3 a 5 años en Puno, Perú; Torres $\mathrm{G}$ et al. (31), mostraron un $20,1 \pm$ 7,4 en niños menores de 71 meses ( 5 años) de edad en Lima, Perú; López R et al. ${ }^{(32)}$, encontró un 17,02 $\pm 10,16$ en niños de 3 a 5 años en Lima, Perú. Otros estudios obtuvieron resultados con menor impacto en la CV en salud oral de los evaluados, ligados en una mayor frecuencia al bajo nivel educativo y cultural por parte de los padres, que consideran que no es necesario tratar los dientes deciduos, y toman como prioridad los dientes permanentes; todo ello, debido al factor económico y la falta de abordaje terapéutico, lo que resulta en perjuicios psicosociales en los niños; Quispe $M$ y Quintana $C{ }^{(14)}$, encontraron un $11,67 \pm 10,15$ en niños de 3 a 5 años en Lima, Perú; López R y García $C$ (16), mostraron un $15,50 \pm 10,24$ en niños de 3 a 5 años en Huaura, Perú; Abanto $\mathrm{J}$ et al. (27), hallaron un 9,21 en niños de 2 a 5 años en Sao Paulo, Brasil; Kramer $P$ et al. (33), identificaron un 0,9 $\pm 3,09$ en niños de 2 a 5 años en Canoas, Brasil; y Díaz $S$ et al. ${ }^{(34)}$, mostraron un 5,05 $\pm 0,45$ en niños de 2 a 5 años en Colombia.

Se debe considerar el impacto del dolor o de las alteraciones funcionales y psicológicas en la CV de un niño. En la sección correspondiente de ECOHIS, las dimensiones que se vieron más afectadas fueron limitación funcional $(6,49 \pm 4,38)$ y autoimagen/ interacción $(2,81 \pm 2,42)$; lo que muestra un impacto negativo en la CV de la salud oral del menor, esto concuerda con Torres $G$ et al. ${ }^{(31)}$, en relación a la dimensión de autoimagen/interacción $(4,7 \pm 1,9)$ y limitación funcional $(4,2 \pm 3,0)$. Otros estudios que difieren respecto a estas puntuaciones; se relacionan al dolor provocado por la CD que, al agravarse, ocasiona disturbios tanto emocionales como físicos, y afectan estas dimensiones con un impacto negativo mayor; por ejemplo, Quispe M y Quintana C (14), resaltan la limitación funcional $(3,09 \pm 3,43)$ y aspecto psicológico $(1,93 \pm 1,89)$ en Lima, Perú; López R y García C (16) en Huaura, Perú, los mismo parámetros con valores distintos, 4,75 $\pm 3,44$ para la limitación funcional y 2,31 $\pm 1,79$ para el aspecto psicológico; López $\mathrm{R}$ et al. (32), limitación funcional $(5,22 \pm 3,42)$ y aspecto psicológico $(2,59 \pm 1,78)$ en Lima, Perú; Kramer et al. ${ }^{(33)}$, limitación funcional $(0,30 \pm 1,16)$ y síntomas orales $(0,17 \pm 0,53)$ en Canoas, Brasil; finalmente, Díaz $\mathrm{S}$ et al. ${ }^{(34)}$, limitación funcional $(1,10 \pm 0,13)$ y síntomas orales $(0,5 \pm 0,05)$.

Al analizar por qué en el Perú los niños presentan alteraciones en limitación funcional, autoimagen/ interacción y aspecto psicológico con gran frecuencia $y$, por lo contrario, en otros países los cambios se observan solo síntomas orales y limitación funcional; encontramos que, en los últimos, existe un mayor interés de los padres por cuidar la dentición decidua $y$, además, cuentan con programas de prevención con una inclusión permanente. Este panorama evidencia que en el Perú hay un descuido del apoderado, y que, cuando la afección se hace crónica y se agrava provoca un impacto sintomatológico y psicosocial en el niño.

Los factores que cumplen un papel importante son la condición económica y el nivel de educación, entre otros, lo que impide buscar y lograr la solución a la afección oral, y esto contribuye con el estrés, preocupación y sensación de culpabilidad del responsable. Por lo tanto, los resultados que se han obtenido, deben generar un análisis mayor, ya que el problema no es parte de un solo individuo, sino que involucra de manera integral a su entorno, es decir, afecta al niño y a su familia. 
La dimensión "angustia de los padres", medida por ECOHIS, con un promedio de $3,72 \pm 2,36$, es similar a lo descrito por Quispe $M$ y Quintana $C{ }^{(14)}$, con un resultado de 2,59 $\pm 1,96$; Torres $G$ et al. (31) $4,3 \pm 1,2$; López $R$ y García C ${ }^{(16)}, 2,71$; López $R$ et al. (32), 2,95 $\pm 1,91$; Kramer et al. (33), $0,18 \pm 0,86$; y Diaz $S{ }^{(34)}, 1,28$; estas cifras reflejan que en todos los estudios encontraron un impacto negativo mayor o menor según sus promedios.

Al relacionar el valor de ECOHIS con la experiencia de $C D$, se encontró un impacto negativo en la $C V$ en salud oral en los niños evaluados; al ser la CD uno de los problemas que involucra, en su cronicidad, al dolor, disfunción, alteración del comportamiento, ausentismo escolar y, además, puede intervenir en las actividades cotidianas y recreativas. Tiene un mayor promedio en el nivel de severidad considerado como muy alto $(30,24$ $\pm 11,11)$ y muestra un $r=0,682$ (Spearman) que es estadísticamente significativo $(p=0,000)$. En diversos estudios también se observa esta relación de manera significativa, como en el trabajo de López R y García $C^{(16)}, 24,88 \pm 9,43(p=0,001)$, en Huaura, Perú; el de Tello $G$ et al. (15), 4,76 ( $p=0,001)$, en Sao Paulo, Brasil; Zambrano 0 et al. (24), 5,81 ( $p=0,001)$ Zulia, Venezuela; Quispe $M$ y Quintana $C{ }^{(14)}, 17,00 \pm 8,93(p=0,001)$ en Lima, Perú; Kramer et al. ${ }^{(33)}, 3,67 \pm 7,52(p=0,001)$ en Canoas, Brasil; y López $R$ et al. ${ }^{(32)}, 18,83 \pm 9,74$ $(p=0,001)$ en Lima, Perú. Las cifras reportadas de significancia en estos estudios reflejan que el índice ceo-d que mide la historia de CD tiene un impacto negativo en la CV en salud oral que afecta a los niños en distintas partes del mundo.

En conclusión, el $88,98 \%$ de casos de $C D$ y el promedio de 6,60 \pm 3,95 del índice ceo-d de la población estudiada refleja el alto riesgo que padece este grupo y muestra los niveles severos de CD. La CV en relación a la salud oral obtuvo un impacto negativo $(21,72 \pm 14,15)$ lo que indica que los niños se ven limitados en sus funciones a nivel oral, y que se alteran la interacción y autoestima del menor; esto confirma que $85,59 \%$ de niños presenta impacto negativo en algún ítem del cuestionario. Existe correlación directa positiva, moderada y significativa entre el índice ceo-d y la CV en la salud oral (CVRSO) de los menores, por lo que, al incrementar el número de $C D$ y su severidad, habrá un mayor impacto negativo en la CV. Esta relación se incrementa con la edad y tiene mayor impacto en las niñas.

\section{Recomendaciones}

Es importante impulsar programas y campañas de prevención y promoción de la salud oral, que cuenten con información adecuada y multidisciplinaria, además de capacitar a los docentes en colegios de bajos recursos para el diagnóstico y tratamiento de caries dental. De esta forma, se evita que este padecimiento se desarrolle en la población vulnerable y que haya una mayor inclusión de estos sectores. Además, es fundamental realizar investigaciones en infantes que incluyan variables como soporte económico y técnico, y que consideren el nivel socioeconómico y el rendimiento familiar.

\section{REFERENCIAS BIBLIOGRÁFICAS}

1. Henostroza G. Caries dental. Principios y procedimientos para el diagnóstico. 1ra ed. Madrid: Ripano; 2007.

2. Elías M, Arellano C. Odontología para bebes. Fundamentos teóricos y prácticos para el clínico. 1ra ed. Madrid: Ripano; 2013.

3. Torres H. Estudio epidemiológico sobre caries dental y necesidades de tratamiento en escolares de 3 a 5 años de edad de huacho, Perú. Revista Salud, Sexualidad y Sociedad. 2010; 3(1):1-4.

4. Villena H. Terapia pulpar. 1ra ed. Lima: Universidad Peruana Cayetano Heredia; 2001.

5. Gruebbel A. Measurement of dental caries prevalence and treatment service for deciduous teeth. Dent Res. 1994; 23:163-168.

6. Duarte I. Análisis de salud oral y su impacto en la calidad de vida de la población preescolar del municipio de Cascais, Portugal, en 2012. [Tesis Doctoral]. Sevilla: Facultad de odontología de la Universidad de Sevilla; 2015.

7. World Health Organization. Oral Health Surveys: Basic Methods. 5th ed. Geneva:WHO; 2013. Disponible en: http:// www.icd.org/content/publications/WHO-Oral-HealthSurveys-Basic-Methods-5th-Edition-2013. pdf

8. Organización Mundial de la Salud. La OMS publica un nuevo informe sobre el problema mundial de las enfermedades bucodentales. Centro de Prensa; Ginebra: OMS; 2004. Disponible en: http://www.who.int/mediacentre/news/ releases/2004/pr15/es/

9. Barnetche M, Cornejo L. Experiencia de caries y calidad de vida de jóvenes en situación de encierro. Rev Salud Pública. 2016; 18(5), 816-826.

10. Porto I, Díaz S. Repercusiones de la salud bucal sobre calidad de vida por ciclo vital individual. Acta Odontológica Colombiana. 2017; 7(2):49-64

11. Organización Mundial de la Salud. ¿Cómo define la OMS la salud? Preguntas más frecuentes; Ginebra:OMS; 2018. Disponible en: http://www.who.int/suggestions/faq/es/

12. Adulyanon $S$, Sheiham A. Oral impacts on daily performances. In: Slade G. Measuring Oral Health of Quality of Life. Chapell Hill: University of North Carolina, Dental Ecology. 1997; 151160. Disponible en: https://www.adelaide.edu.au/arcpoh/ downloads/publications/reports/miscellaneous/measuringoral-health-and-quality-of-life.pdf

13. Chaustre L, Calderón L, Saucedo V. Calidad de vida relacionada con la salud oral en preescolares vinculados con el instituto colombiano de bienestar familiar de Bucaramanga según la versión peruana del Early Childhood Oral Health Impact Scale. [Tesis de Titulación]. Bucaramanga: 
Universidad Santo Tomás; 2016. Disponible en: http:// repository.usta.edu.co/bitstream/handle/11634/1698/2016ChaustreTorres\%2CLeinnsyKarina-Trabajodegrado. pdf?sequence $=1$ \&isAllowed $=y$

14. Quispe M, Quintana C. Impacto de la salud oral sobre la calidad de vida en niños preescolares de la institución educativa "José Antonio Encinas" N. ${ }^{\circ} 1137$ en el distrito de Santa Anita, Lima. Revista de Investigación de la Universidad Norbert Wiener. 2017; (6): 61-71.

15. Tello G, Abant J, Butini L, Murakami C, Bonini G, Bönecker M. Impacto de los principales problemas de salud bucal en la calidad de vida de preescolares. Revista Odontología. 2016; 19(2):42-52.

16. López R, Garcia C. Calidad de vida y problemas bucales en preescolares de la provincia de Huaura, Lima. Rev Estomatol Herediana. 2014; 23(3):139-47. Disponible en: http://www. upch.edu.pe/vrinve/dugic/revistas/index.php/REH/article/ viewFile/24/17.

17. Bordoni N, Claravino O, Zambrano O, Villena R, Beltran E, Squassi. Early Childhood Oral Health Impact Scale (ECOHIS): Translation and validation in spanish language. Acta Odontol Latinoam. 2012; 35(3):270-278.

18. Hernández R, Fernández $C$, Baptista P. Metodología de la investigación. 6ta ed. México: McGraw-Hill; 2014: 634pp Disponible en: https://periodicooficial.jalisco.gob.mx/sites/ periodicooficial.jalisco.gob.mx/files/metodologia_de_la_ investigacion_-_roberto_hernandez_sampieri.pdf

19. Ley que modifica, adiciona y deroga diversos artículos de La Ley $N^{\circ} 15251$, ley que crea el Colegio Odontológico del Perú - Congreso De La República. 2007. Disponible en: http: / / www.leyes.congreso.gob.pe/Documentos/2016_2021/ADLP/ Normas_Legales/30699-LEY.pdf

20. Colegio Odontológico del Perú. Normas técnicas del Odontograma. Perú: Consejo Administrativo Nacional; 2015. Disponible en: http://www.cop.org.pe/wp-content/ uploads/2015/05/Normas-tecnicas-del-Odontograma.pdf

21. Mazzetti P, Zorrilla H, Podestá L. Manual de Seguridad. Programa Nacional de Hemoterapia y Bancos de Sangre. Lima: 015 ${ }^{\circ}$ Ministerio de Salud; 2004.

22. Bansal P, Sujlana A, Pannu P, Kour R. Dental Discomfort Questionnaire: correlated with clinical manifestations of advanced dental caries in young children. J Dent Specialities. 2017; 5(1):3-7.

23. Alosaimi B, Alturki G, Alnofal S, Alosaimi N, Ansari S. Assessing untreated dental caries among private and public preschool children in Riyadh, a cross-sectional study design. J Dent Oral Health. 2017; 10(3).

24. Zambrano O, Fong L, Rivera L, Calatayud E, Hernández J, Maldonado A, et al. Impacto de la caries de infancia temprana en la calidad de vida del niño zuliano y su familia. Odous Científica. 2015; 16(2):8-17.

25. Bagińska J, Rodakowska E, Wilczyńska M, Jamiołkowski J. Index of clinical consequences of untreated dental caries (pufa) in primary dentition of children from north-east Poland. 2013; 58(2):442-447.

26. Kamran R, Farooq W, Riaz F, Jahangir F. Clinical consequences of untreated dental caries assessed using PUFA index and its covariates in children residing in orphanages of Pakistan. BMC Oral Health. 2017; 17:108.

27. Abanto J, Carvalho T, Mendes F, Wanderley M, Bönecker M, Raggio D. Impact of oral diseases and disorders on oral health-

related quality of life of preschool children. Community Dent Oral Epidemiol. 2011; 39(2):105-114.

28. Patil S, Hathiwala S. Kumar D, Raj S, Khatri S, Singh A. Impact of untreated dental caries on school attendance among the preschool children. Int J Pharm Pharm Res. 2016; 8(2):570572.

29. Mehta A, Bhalla A. Assessing consequences of untreated carious lesions using pufa index among 5-6 years old school children in an urban Indian population. Indian J Dent Res. 2014; 25(2):150-153.

30. Mamani V y Padilla T (2016), Riesgo estomatológico e impacto de las afecciones bucales en la calidad de vida de preescolares del ámbito rural en Puno. Odontol Pediatr. 2015; 14 (2): 108115.

31. Torres G, Blanco D, Chávez M, Apaza S, Antezana V. Impacto de la caries de la infancia temprana en la calidad de vida relacionada a la salud bucal en niños peruanos menores de 71 meses de edad. Odontol Sanmarquina. 2015; 18(2):87-94.

32. López R, García, C, Villena R, Bordoni N. Cross cultural adaptation and validation of the Early Childhood Health Impact Scale (ECOHIS) in peruvian preschoolers. Acta Odontol Latinoam. 2013; 26 (2), 60-67.

33. Kramer P, Feldens C, Ferreira S, Bervian J, Rodrigues P, Peres $M$. Exploring the impact of oral diseases and disorders on quality of life of preschool children. Community Dent Oral Epidemiol. 2013; 41(4):327-335.

34. Díaz S, Gonzales F, Ramos K, Pérez S, Malo L. Impacto de caries dental sobre la calidad de vida en niños pertenecientes a la primera infancia de instituciones educativas de Cartagena. [Tesis Doctoral]. Cartagena: Facultad de Odontología de Cartagena; 2015.

Fuentes de financiamiento:

Este artículo ha sido financiado por el Doctor Joel Maycol Rocha Lerzundi.

\section{Conflictos de interés:}

Los autores declaran no tener ningún conflicto de interés.

\section{Correspondencia:}

Joel Maycol Rocha Lerzundi

Dirección: Psje.de la Perspectiva Edif.101 Dpto.202, Dammert Muelle, Surquillo. Lima, Perú.

Teléfono: (+51) 972542818 - 964396691.

Correo electrónico: schester27@hotmail.com

Recibido: 25 de agosto de 2018.

Evaluado: 04 de setiembre de 2018 Aprobado: 16 de octubre de 2018.

(c) La revista. Publicado por Universidad de San Martín de Porres, Perú. (cc) Ву Licencia de Creative Commons Artículo en acceso abierto bajo términos de Licencia Creative Commons Atribución 4.0 Internacional. (http: //creativecommons.org/licenses/by/4.0/)

\section{ORCID IDS}

Rocha Lerzundi Joel Maycol Gómez Gonzales Walter Edgar Bernardo Santiago Grisi https://orcid.org/0000-0002-6175-9980 https://orcid.org/0000-0003-0706-7614 https://orcid.org/0000-0003-0977-1349 